\title{
Reproductive Health and Child Health Profile in the Field Practice Area of RajaRajeswari Medical College and Hospital, Bengaluru: A Descriptive Study
}

\author{
${ }^{1}$ Shashikala Manjunatha, ${ }^{2} \mathrm{KS}$ Ramya
}

\begin{abstract}
Introduction: Reproductive health, child health and nutrition are core priorities for any country, more so for India, with the world's greatest burden of maternal, newborn, and child deaths.

Objectives: To assess the reproductive health and child (under 5 years) health profile of the defined population using reproductive health and child health indicators in the field practice area of RajaRajeswari Medical College and Hospital.
\end{abstract}

Materials and methods: A community-based cross-sectional study was conducted among women of reproductive age group (15-49) using a pretested and semistructured questionnaire which included reproductive health and child health indicators.

Method of analysis: Data ware analyzed using Statistical Package for Social Sciences (SPSS) software.

Results: Among 658 mothers, $60 \%$ were using family planning methods, $63 \%$ of mothers had full antenatal check-up and $80 \%$ had institutional delivery. Around $80 \%$ of the children between 12 and 23 months were fully immunized, $60 \%$ of children were exclusively breastfed. Ninety-two percent of the women had heard of HIVIAIDS.

Conclusion: Awareness and utilization of various reproductive and child health services among the women of reproductive age group was found to be far from satisfactory and, hence, there is a need to raise the awareness in this regard through effective information, education and communication (IEC) activities.

Keywords: Bengaluru, Child health, Reproductive and child health indicators, Reproductive health.

How to cite this article: Manjunatha S, Ramya KS. Reproductive Health and Child Health Profile in the Field Practice Area of RajaRajeswari Medical College and Hospital, Bengaluru: A Descriptive Study. J Med Sci 2015;1(2):27-31.

Source of support: Nil

Conflict of interest: None

\footnotetext{
${ }^{1}$ Professor and Head, ${ }^{2}$ Postgraduate

1,2Department of Community Medicine, RajaRajeswari Medical College and Hospital, Bengaluru, Karnataka, India
}

Corresponding Author: Shashikala Manjunatha, Professor and Head, Department of Community Medicine RajaRajeswari Medical College and Hospital, Mysore Road Bengaluru-74, Karnataka, India, Phone: 08028437444, e-mail: manjunathashashikala@gmail.com

\section{INTRODUCTION}

Reproductive and Child Health $(\mathrm{RCH})$ has been defined as a state in which 'People have the ability to reproduce and regulate their fertility; women are able to go through pregnancy and childbirth safely, the outcome of pregnancy is successful in terms of maternal and infant survival and well-being, and couples are able to have sexual relations free of the fear of pregnancy and contracting diseases.'

The estimated maternal mortality ratio showed a $36 \%$ reduction from 398 per 100000 live births in 1997 to 2008 to 254 per 100000 live births in 2004 to 2006 . However, this decrease is not sufficient to achieve a maternal mortality ratio of less than 100 per 100000 live births to meet national goals or the Millennium Development Goal (MDG) 5 by $2015 .^{2}$

The mortality rate for children (age $<5$ years) in India was 63 per 1000 live births in 2010. ${ }^{3}$ At the current rate of progress, India will not be able to achieve the MDG 4 target of 38 per 1000 live births by 2015. In 2008, infant mortality rate in India was 53 per 1000 live births. The national goal is to attain a rate of less than 30 per 1000 live births by 2010 . More than $48 \%$ of children (age $<5$ years) are stunted (height for age $<-2 \mathrm{Z}$ score), $43 \%$ are underweight (weight for age $<-2 \mathrm{Z}$ score), and about $20 \%$ have wasting (weight for height $<-2 \mathrm{Z}$ score). At the current rate of decline, India will not achieve the national goals for child nutrition or the MDG 1 target for child nutrition, which is to reduce the prevalence of underweight among children (age $<5$ years) to $27 \%$ by 2015. ${ }^{4}$

The nutritional status of Indian women is inadequate: $33 \%$ of married women (15-49 years) are too thin (i.e. BMI $<18.5 \mathrm{~kg} / \mathrm{m}^{2}$ ), and $11 \%$ are too short (i.e. height $<145 \mathrm{~cm})^{3}$ In 2004 , more than $75 \%$ of women were anemic. $^{5}$ The reported prevalence of anemia in pregnant women from large-scale surveys range from $74.3 \%$ (22) to $96.2 \%$ (23). Added to these burdens are the challenges of the epidemiological transition. Overnutrition is emerging as an important public health problem-12.6\% of women and $9.3 \%$ of men aged 15 to 49 years have a body mass index (BMI) of $25 \mathrm{~kg} / \mathrm{m}^{2}$ or more, and is more common among people living in urban areas. ${ }^{2}$ 
Steady declines have been noted in fertility, maternal, infant and child mortalities, and the prevalence of severe manifestations of nutritional deficiencies, but the pace has been slow and falls short of national and MDG targets. The likely explanations include social inequities, disparities in health systems between and within states, and consequences of urbanization and demographic transition. India has diverse social, demographic, economic, geographic, and health systems. Wide differentials exist across states in the indicators for reproductive health, and child health and nutrition. ${ }^{2}$

The leading causes of maternal deaths in India are hemorrhage, sepsis, complications of abortion, and hypertensive disorders. ${ }^{6}$ More than half of deaths in children (age $<5$ years) occur in the neonatal period; infections (including sepsis, pneumonia, diarrhea, and tetanus), prematurity, and birth asphyxia are the three major causes of death in this period. The remaining $45 \%$ of deaths occur in children aged between 1 month and 59 months, and the major causes are pneumonia and diarrhea. ${ }^{7}$

The immediate causes of poor reproductive health, and child health and undernutrition have underlying social, economic, and environmental determinants: literacy, women's status, sociocultural beliefs, caste, taboos and above all, income level. Analysis of data from National Family Health Survey (NFHS)-3 showed that illiteracy, low wealth index, rural habitat, and perceived small size at birth were significantly associated with neonatal and infant mortality. For low birth weight, maternal age at childbirth of less than 20 years was associated with a roughly $50 \%$ excess risk compared with childbirth at older age. Antenatal care seemed to protect strongly against low birth weight. These analyses indicate the importance of delaying childbirth until after 20 years of age, and providing antenatal care and birth spacing as interventions to improved child survival. ${ }^{2}$

For rendering the above stated services, the new approach under the $\mathrm{RCH}$ program places emphasis on client-oriented, need-based, high quality, integrated services to the beneficiaries. ${ }^{8}$ Healthy pregnant women give birth to healthy children who grow into healthy adolescents. Good health during adolescent years leads to health during reproductive years. The cycle continues into the next generation when a healthy pregnant ensures a healthy child. If implemented in an integrated manner, $\mathrm{RCH}$ will go a long way toward improving the overall health of women and that of society as a whole. ${ }^{9}$

India has not achieved coverage of more than $55 \%$ for any of the priority interventions for reproductive health, and child health and nutrition. For example, only $46 \%$ of infants were exclusively breastfed in the first 6 months of life in 2007 to 2008 , and just $24 \%$ received solid or semisolid feeds and breastfeeds at 6 to 9 months. ${ }^{10}$

Research would, therefore, be needed to, e.g. to formulate strategies to build rational and healthy sexual attitude and behavior among adolescents and youths, approaches to ending discrimination and injustice, better understanding of barriers to girls education, empowerment and development, improve men's participation in reproductive healthcare needs.

Hence, the study was carried out in the field practice area of Department of Community Medicine, RajaRajeswari Medical College and Hospital, Bengaluru, with the objective to assess the reproductive health and child (under 5 years) health profile of the defined population using reproductive health and child health indicators.

\section{MATERIALS AND METHODS}

A cross-sectional descriptive study was conducted for 1 year among all women of reproductive age group (15- 49 years) and under-five children in the field practice area of RajaRajeswari Medical College and Hospital. Considering the least prevalence of $7.9 \%$ of pregnancy induced hypertension according to DLHS-3, with $15 \%$ allowable error, the estimated sample size for the study using the formula $4 \mathrm{pq} / \mathrm{L}^{2}$ is 2590 . Data were collected through interview method. A pretested and semistructured questionnaire was used for the assessment of reproductive and child health using a checklist based on reproductive health and child health indicators. The data were collected using purposive sampling method. Institutional Ethical Committee approval was obtained prior to initiation of study. Written informed consent was taken from all study subjects before data collection, from the parents or care takers in case of under-five children. The housing conditions were assessed during the house visit. Awareness about government health programs were based on response from households. Data on antenatal care were based on women whose last pregnancy outcome was live/still birth during the reference period. Child feeding practices were assessed for the last born child in the household. Data on treatment practices for childhood diseases were collected based on last two children born during the reference period. Data were analyzed using Statistical Package for Social Sciences (SPSS) software.

\section{RESULTS}

Among the 1014 households, 96\% had electricity, 76\% had access to toilet facility, $14 \%$ live in kachcha houses 
and $45 \%$ live in pucca houses and $94 \%$ had improved sources of drinking water as shown in Table 1. It was observed that $82.7 \%$ of the study subjects above 7 years were literate. The mean age of marriage for boys was 27 years and that for girls was 21 years.

\section{AWARENESS ABOUT GOVERNMENT HEALTH PROGRAMS}

It was found that $96 \%$ of the study subjects were aware of health programs on malaria, dengue and chikungunya, $83 \%$ had awareness regarding prevention of sex selection during antenatal scanning, 79\% on directly observed treatment short-course (DOTS) treatment for tuberculosis and $74 \%$ were aware about the health program on leprosy eradication.

\section{Fertility}

It was observed that the mean number of children ever born to women aged between 40 and 44 years was 3.7. The birth order of two and above among women aged 20 to 24 years was $54 \%$. Out of total births, $45 \%$ of births were to women during the age 15 months to 19 years. The $40 \%$ women with two children did not want any more children.

Table 1: Demographic profile of the study subjects

\begin{tabular}{ll}
\hline Access to electricity & $96 \%$ \\
Access to toilet facility & $76 \%$ \\
Kachcha houses & $14 \%$ \\
Pucca houses & $45 \%$ \\
Access to improved source of drinking water & $94 \%$ \\
Population literate above 7 years & $82.7 \%$ \\
Girls married below age 18* & $7 \%$ \\
Boys married below age 21* & $2 \%$ \\
Currently married women of the age 20 to 24 years & $24 \%$ \\
who were married before the age of 18 years & \\
\hline
\end{tabular}

*Marriages that occurred during the reference period

\section{Current Use of Family Planning Methods}

It was found that $60 \%$ of the study subjects were using family planning (any method), 50\% women had underwent female sterilization, $0.5 \%$ male sterilization, $0.1 \%$ were using pills, $3.8 \%$ were using intrauterine devices, $4 \%$ were using condoms and $1.9 \%$ following rhythm method. The $0.9 \%$ had used emergency contraceptive pills.

\section{AWARENESS OF CONTRACEPTIVE METHODS}

About $62 \%$ of the unmarried women knew about condom, $78 \%$ of them knew about oral contraceptive pills and 55\% on them knew about emergency contraceptive pills.

\section{Antenatal Care}

It was found that $96 \%$ of the mothers received any antenatal care, $86 \%$ of mothers had antenatal check-up in the first trimester, $91 \%$ of the mothers had more than three antenatal check-ups. The $93 \%$ of the mothers had at least one tetanus toxoid injection. The $66 \%$ of them consumed 100 Iron and Folic acid tablets as shown in Graph 1. The $63 \%$ of the mothers had full antenatal check-up. The $80 \%$ of them had institutional deliveries.

\section{Child Immunization}

Among 478 under-five children, $80 \%$ of children between 12 and 23 months, were fully immunized and $0.5 \%$ of children in this age group had not received any vaccine.

\section{Child Feeding Practices}

It was observed that $49 \%$ of the children less than 3 years of age were breastfed within 1 hour of birth, $60 \%$ of the children between 0 and 6 months were exclusively breastfed, 38\% of children between 6 and 35 months were exclusively breastfed for at least 6 months. Among

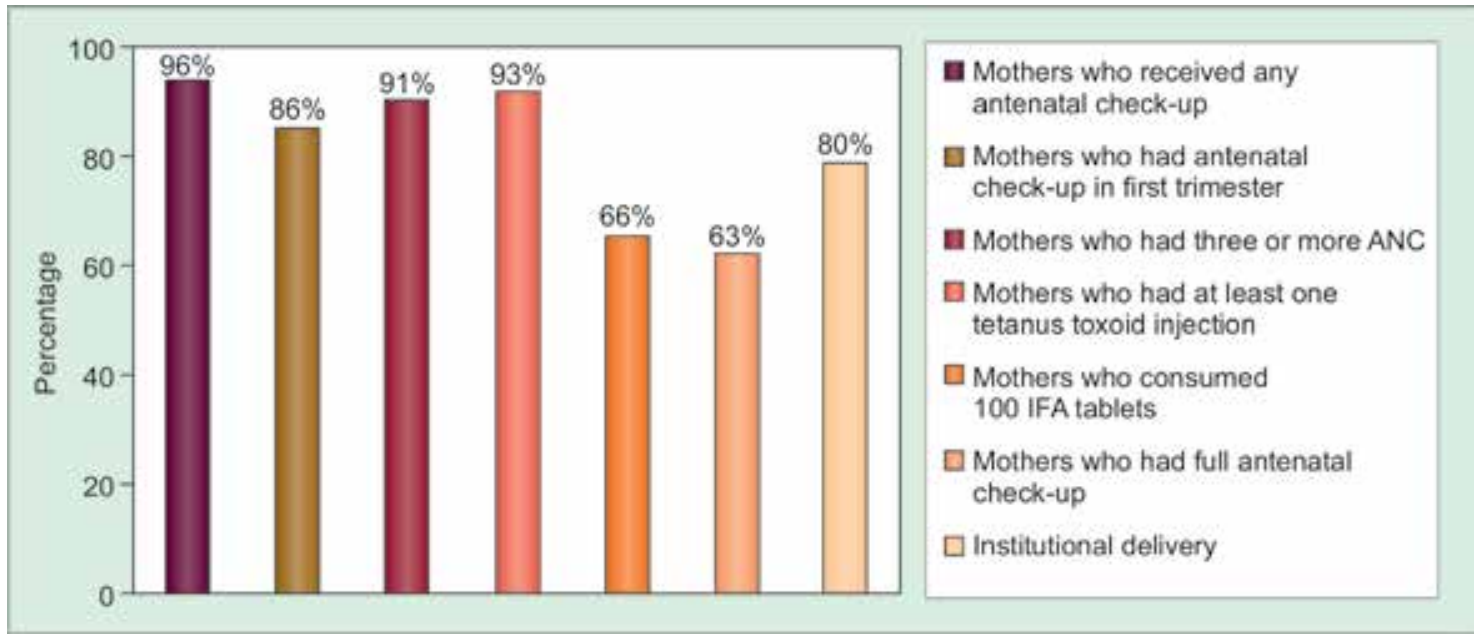

Graph 1: Antenatal care among mothers 


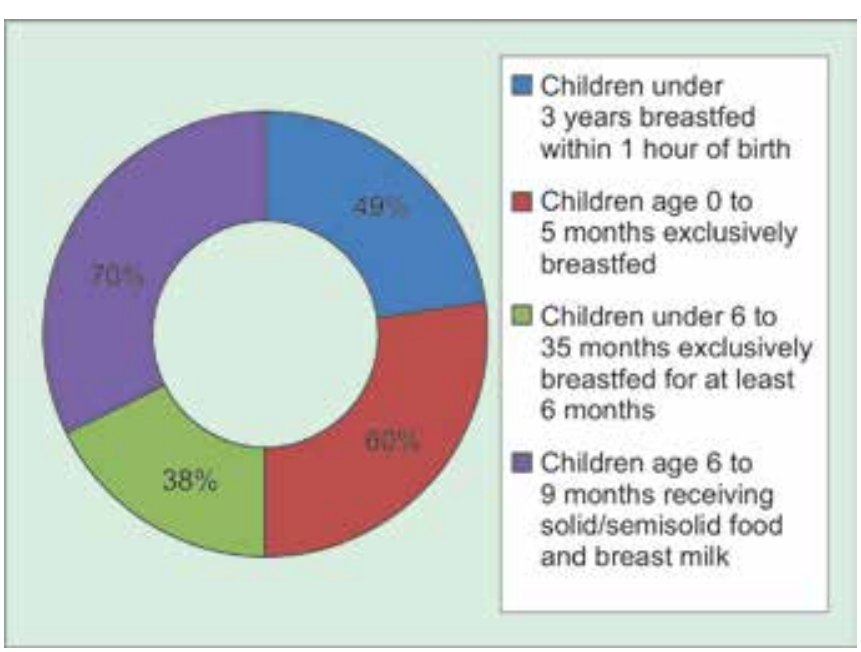

Graph 2: Doughnut graph showing child feeding practices

children between 6 and 9 months, $70 \%$ of them received solid/semisolid food and breast milk as shown in Graph 2.

\section{Treatment of Childhood Diseases}

It was observed that $52 \%$ of the under-five children with diarrhea in the last 2 weeks received oral rehydration salts (ORS), $81 \%$ of them with diarrhea in the last 2 weeks sought treatment, $84 \%$ of children with acute respiratory tract infection (ARI) or fever in last 2 weeks sought treatment or advice and around $45 \%$ of the women were aware of the danger signs of ARI as shown in Graph 3.

\section{Awareness of Reproductive Tract Infection/Sexually Transmitted Infections and HIVIAIDS}

Majority of the women $92 \%$ had heard of human immunodeficiency virus/acquired immune deficiency syndrome (HIV/AIDS), $42 \%$ of them had heard of reproductive tract infection/sexually transmitted infections (RTI/STI). Around 16\% of the women had any symptoms of RTI/STI, $81 \%$ of them knew the place to go for testing of HIV/AIDS and $36 \%$ of them had underwent HIV/AIDS testing.

\section{DISCUSSION}

In the present study, it was found that $24 \%$ of currently married women were married before the age of 18 years as compared to $22.1 \%$ of women getting married below 18 years at the national level as per NFHS-3 reports.

In the present study, it was found that the awareness of RTI/STI was $42 \%$ and presence of any symptoms of RTI/STI is $16 \%$. In a study conducted by Rizwan et al, ${ }^{11}$ it was found that the awareness regarding RTI/STI was $56.4 \%$ and prevalence of any RTI symptom was $36.3 \%$.

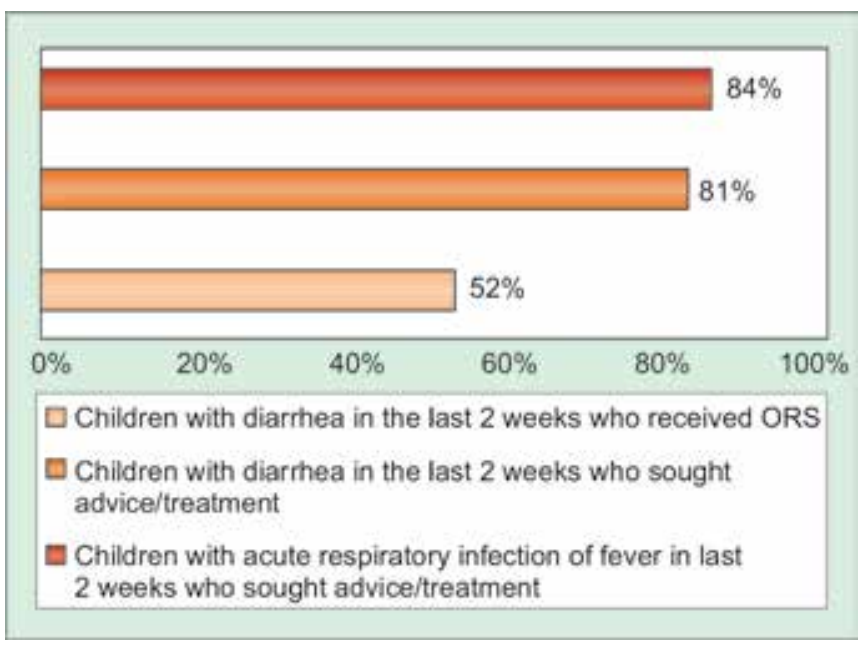

Graph 3: The treatment of childhood diseases

In the study, it was observed that the current use of any family planning method was $60 \%$ among the women which is comparable to $56.3 \%$ contraceptive use among women according to NFHS-3 report.

It was found that $60 \%$ of the children between the ages 0 and 6 months of age were exclusively breastfed and $70 \%$ of the children aged 6 and 9 months received solid/ semisolid food and breast milk, whereas the DLHS-3 (2007 and 2008) report showed that $46 \%$ of infants were exclusively breastfed in the first 6 months of life and just $24 \%$ received solid and semisolid feeds and breastfeeds at 6 to 9 months. A wide variation exists between states and across districts. ${ }^{10}$

\section{CONCLUSION}

It can be concluded from the present study that the awareness regarding health programs related to reproductive and child health, family planning methods, antenatal care, breastfeeding practices, treatment for childhood diseases, reproductive tract infections and sexually transmitted infections is far from satisfactory.

\section{RECOMMENDATIONS}

It is recommended to raise the awareness about reproductive health and child health among the women of reproductive age through information, education and communication (IEC) activities by collaborating with local nongovernmental organizations, thereby building public private partnerships in improvement of utilization of $\mathrm{RCH}$ services.

\section{ACKNOWLEDGMENTS}

We thankfully acknowledge the support of the study participants, Dr Swapna, Dr Padma, Medical Officer of Urban Health Training Center, urban field practice area, Mr Sampath Kumar, Medical Social Worker and 
the team of the Department of Community Medicine, RajaRajeswari Medical College and Hospital, in conducting the study.

\section{REFERENCES}

1. Park K. Park's Textbook of Preventive and Social Medicine. 22nd ed. Jabalpur: Banaridas Bhanot; 2013.

2. Paul VK, Sachdev HS, Mavalankar D, et al. Reproductive health, and child health and nutrition in India: meeting the challenge. Lancet 2011;377:332-349.

3. Rajaratnam JK, Marcus JR, Flaxman AD, et al. Neonatal, postneonatal, childhood, and under-5 mortality for 187 countries, 1970-2010: a systematic analysis of progress towards Millennium Development Goal 4. Lancet 2010;375: 1988-2008.

4. World Bank. India's undernourished children-a call for reform and action. World Bank: Washington DC; 2006.

5. National Nutrition Monitoring Bureau (NNMB). NNMB Micronutrient survey. Hyderabad: National Institute of Nutrition; 2002.
6. Registrar General of India. Special bulletin on maternal mortality 2004-2006. New Delhi: Registrar General of India; 2009.

7. Black RE, Cousens S, Johnson HL, et al. Global, regional, and national causes of child mortality in 2008: a systematic analysis. Lancet 2010;375:1969-1987.

8. Indian Public Health Association. Reproductive and Child Health Workshop [home page on the internet] [cited on 2015 Jan 6]. Available at: http://www.iphaonline.org/news/ reports/pdf/report_rch_workshop.pdf.

9. Kishore J. National Health Programs of India. 11th ed. New Delhi: Century Publications; 2014. p. 141.

10. IIPS. District Level Household Survey (DLHS-3), India 2007-2008: fact sheet. Mumbai: International Institute for Population Sciences; 2008.

11. Rizwan SA, et al. KAP study on sexually transmitted infections/reproductive tract infections among married women in rural Harayana. Indian Dermatol Online J 2015; 6(1):9-12. 